\title{
Fundamental equivalence of discrete-time AR representations
}

\author{
A.I.G. Vardulakis, E.N. Antoniou \\ Department of Mathematics \\ Aristotle University of Thessaloniki \\ 54006 - Thessaloniki -GREECE \\ Tel: +3031997951 - Fax: +3031997951 \\ E-mail: avardula@ccf.auth.gr antoniou@ccf.auth.gr.
}

May 7, 2003

\begin{abstract}
We examine the problem of equivalence of discrete time auto-regressive (AR) representations. Two AR representations are defined as fundamentally equivalent if their solution spaces or behaviors are isomorphic. Starting from the fact that the behavior of an AR representation, when considered over a finite time interval, depends on the algebraic structure of both the finite and the infinite elementary divisors of the underlying polynomial matrix we examine closely this structure and show that it can be easily exposed through the corresponding structure of a block companion matrix pencil which can be easily constructed from the coefficients of the original matrix. As a consequence the proposed block companion matrix pencil constitutes the natural first-order fundamentally equivalent representation (realization) of such an AR representation. As a further consequence and generalization we show that two AR representations described by polynomial matrices of possibly different degrees and dimensions that give rise to fundamentally equivalent first-order representations are fundamentally equivalent.
\end{abstract}

\section{Introduction}

Consider a continuous time and free scalar linear system governed by an $n-t h$ order homogeneous differential equation

$$
a(\rho) \xi(t)=0, \quad t \geq 0
$$

where $\xi(t):[0, \infty) \rightarrow \mathbb{R}, \rho$ is the differential operator and $a(\rho):=a_{n} \rho^{n}+$ $a_{n-1} \rho^{n-1}+\ldots+a_{1} \rho+a_{o} \in \mathbb{R}[\rho]$. It is then well known that if we define the 
matrices

$E:=\left[\begin{array}{ccccc}1 & 0 & 0 & \ldots & 0 \\ 0 & 1 & 0 & \ldots & 0 \\ \vdots & \vdots & \vdots & \ldots & \vdots \\ 0 & 0 & 0 & \ddots & 0 \\ 0 & 0 & 0 & \ldots & a_{n}\end{array}\right] \in \mathbb{R}^{n \times n}, A:=\left[\begin{array}{lllll}0 & 1 & 0 & \ldots & 0 \\ 0 & 0 & 1 & \ldots & 0 \\ \vdots & \vdots & \vdots & \ddots & \vdots \\ 0 & 0 & 0 & \ldots & 1 \\ -a_{0} & -a_{1} & -a_{2} & \ldots & -a_{n-1}\end{array}\right] \in \mathbb{R}^{n \times n}$

and the vector

$$
\mathbf{x}(t)=\left[\begin{array}{l}
x_{1}(t) \\
x_{2}(t) \\
\vdots \\
x_{n}(t)
\end{array}\right]:=\left[\begin{array}{l}
\xi(t) \\
\rho \xi(t) \\
\vdots \\
\rho^{n-1} \xi(t)
\end{array}\right]
$$

then system (1) can be described by $n$ first order differential equations represented in matrix form by

$$
E \rho \mathbf{x}(t)=A \mathbf{x}(t), \quad t \geq 0
$$

and the 'matrix pencil'

$$
s E-A=\left[\begin{array}{lllll}
s & -1 & 0 & \ldots & 0 \\
0 & s & -1 & \ldots & 0 \\
\vdots & \vdots & s & \ddots & \vdots \\
0 & 0 & 0 & \ldots & -1 \\
a_{0} & a_{1} & a_{2} & \ldots & s a_{n}+a_{n-1}
\end{array}\right] \in \mathbb{R}[s]^{n \times n}
$$

has the same (finite) elementary divisors and thus the same (finite) zeros as the characteristic polynomial $a(s)$ of (1) i.e. the Smith form $S_{s E-A}^{\mathbb{C}}$ in $\mathbb{C}$ of $s E-A$ is given by

$$
S_{s E-A}^{\mathbb{C}}=\operatorname{diag}[1,1, \ldots, 1, a(s)]
$$

Furthermore if $a(s)$ is factored as

$$
a(s)=\left(s-\lambda_{1}\right)^{m_{1}}\left(s-\lambda_{2}\right)^{m_{2}} \cdots\left(s-\lambda_{l}\right)^{m_{l}}
$$

where $\lambda_{i} \in \mathbb{C}, i=1,2, \ldots, l$ are the distinct zeros of $a(s)$ and $m_{1}+m_{2}+\ldots+m_{l}=$ $n$,

$$
J_{F}:=\text { blockdiag }\left[J_{1}, J_{2}, \ldots, J_{l}\right] \in \mathbb{R}^{n \times n}
$$

where

$$
J_{i}:=\left[\begin{array}{lllll}
\lambda_{i} & 1 & 0 & \ldots & 0 \\
0 & \lambda_{i} & 1 & \ldots & 0 \\
\vdots & \vdots & \ddots & \ddots & \vdots \\
0 & 0 & \ldots & \lambda_{i} & 1 \\
0 & 0 & \ldots & 0 & \lambda_{i}
\end{array}\right] \in \mathbb{R}^{m_{i} \times m_{i}}, \quad i=1,2, \ldots, l
$$

and $C_{F} \in \mathbb{R}^{n \times n}$ is such that the pair $\left(C_{F}, J_{F}\right)$ is a (finite) Jordan pair of $s E-A$ $[10][13]$ i.e. if

$$
E C_{F} J_{F}=A C_{F} \text { and } \operatorname{rank}_{\mathbb{R}}\left[\begin{array}{l}
C_{F} \\
C_{F} J_{F} \\
\vdots \\
C_{F} J_{F}^{n-1}
\end{array}\right]=n
$$


then the solution of (4) is given by

$$
\mathbf{x}(t)=C_{F} e^{J_{F} t} \mathbf{x}(0-), \quad t \geq 0
$$

where

$$
\mathbf{x}(0-)=\left[\begin{array}{l}
x_{1}(0-) \\
x_{2}(0-) \\
\vdots \\
x_{n}(0-)
\end{array}\right]=\left[\begin{array}{l}
\xi(0-) \\
\xi^{(1)}(0-) \\
\vdots \\
\xi^{(n-1)}(0-)
\end{array}\right]
$$

and $\xi^{(i)}(t):=\frac{d^{i} \xi(t)}{d t^{i}}$ and thus the solution of (1) is given by

$$
\xi(t)=x_{1}(t)=\underbrace{\left[\begin{array}{llll}
1 & 0 & \ldots & 0
\end{array}\right]}_{n} \mathbf{x}(t)
$$

so that systems (1) and (4) are fundamentally equivalent [18].

The generalization of the above simple ideas to the multivariable case

$$
A(\rho) \xi(t)=0, \quad t \geq 0
$$

where $\xi(t):[0, \infty) \rightarrow \mathbb{R}^{r}$ is a vector valued function and $A(\rho)=A_{q} \rho^{q}+$ $A_{q-1} \rho^{q-1}+\ldots+A_{0} \in \mathbb{R}[\rho]^{r \times r}, A_{k} \in \mathbb{R}^{r \times r}, k=0,1, \ldots, q \geq 1, A_{q} \neq 0, \operatorname{det} A_{q} \geq 0$ has been presented in [10] through the formation of the block companion matrix pencil

$$
\rho \bar{E}-\bar{A}:=\left[\begin{array}{ccccc}
\rho I_{r} & -I_{r} & \ldots & 0 & 0 \\
0 & \rho I_{r} & \ldots & 0 & 0 \\
\vdots & \vdots & \ddots & \vdots & \vdots \\
0 & 0 & \ldots & \rho I_{r} & -I_{r} \\
A_{0} & A_{1} & \ldots & A_{q-2} & \rho A_{q}+A_{q-1}
\end{array}\right] \in \mathbb{R}[\rho]^{r q \times r q}
$$

and the introduction of the vector $x(t):=\left[\begin{array}{llll}\xi(t)^{\top} & \rho \xi(t)^{\top} & \ldots & \rho^{q-1} \xi(t)^{\top}\end{array}\right]$ : $[0, \infty) \rightarrow \mathbb{R}^{r q}$ but the exact relation between the finite and infinite zero structures of $A(\rho)$ and $\rho \bar{E}-\bar{A}$ in the sense of their Smith-Mcmillan forms in $\mathbb{C}$ and at infinity was not examined there. The exact relation between the SmithMcMillan finite and infinite zero structures of $A(\rho)$ and what it turns out to be $^{1}$ the Wierstrass canonical form [9] of $\rho \bar{E}-\bar{A}$ was examined in [16][15][13] (chapter 4, section 4.2.4) where the effect of the singularity of $A_{q}$ on the zero structure at infinity of $A(\rho)$ and the impulsive behavior of $\xi(t)$ at $t=0$ was also investigated. Based on the results in [18] it can be proved that systems $A(\rho) \xi(t)=0$ and $[\rho \bar{E}-\bar{A}] x(t)=0$ are fundamentally equivalent.

In this paper we examine the implications of the above ideas in the multivariable discrete time case and apply them to the problem of equivalence of discrete time auto-regressive (AR) representations.

We firstly review the fact that although in the continuous time case the infinite elementary divisors (see section 2 bellow) of the polynomial matrix $A(\rho)$ do not have a direct dynamic interpretation on the behavior or solution space of an AR representation as in (6) this is not so in the case of discrete time

\footnotetext{
${ }^{1}$ as shown in Lemma 8 bellow.
} 
AR representations. Recent results [7][17] show that when the discrete time AR representation

$$
A(\sigma) \xi_{k}=0
$$

(where $\sigma$ denotes the forward shift operator $\sigma^{i} \xi_{k}=\xi_{k+i}$ ) is considered over a finite time interval ${ }^{2} k=0,1,2, \ldots, N$ then its behavior depends on both the finite and the infinite elementary divisors of the corresponding polynomial matrix $A(\sigma)$ and it is causal iff $A(\sigma)$ has no infinite elementary divisors. We next examine the relation of the algebraic structure of the finite and infinite elementary divisors of the nonsingular polynomial matrix $A(\sigma)$ and the corresponding structure of the matrix pencil $\sigma \bar{E}-\bar{A}$ as in (7). As mentioned above the block companion form of this matrix pencil has been studied in the past but only from the finite elementary divisor and finite zero point of view. In this paper we extend these results to the point at infinity. In particular, it is shown that the structure of both the finite and the infinite elementary divisors of a square - invertible polynomial matrix $A(\sigma)$ is isomorphic to the corresponding structure of the matrix pencil $\sigma \bar{E}-\bar{A}$. A consequence of this result is that the discrete time AR-representations associated with $A(\sigma)$ and $\sigma \bar{E}-\bar{A}$ have isomorphic behaviors and the one associated with $\sigma \bar{E}-\bar{A}$ constitutes the natural first order equivalent realization of the homogeneous discrete time $\mathrm{AR}$ representation in (8). Finally and as an other consequence and generalization of our results we examine the notion of fundamental equivalence of discrete time AR representations described by polynomial matrices of different degrees and dimensions.

\section{Background}

In what follows $\mathbb{R}, \mathbb{C}$ denote respectively the fields of real and complex numbers and $\mathbb{Z}, \mathbb{Z}^{+}$denote respectively the integers and non negative integers. By $\mathbb{R}(\sigma)^{p \times m}, \mathbb{R}_{p r}(\sigma)^{p \times m}$ and $\mathbb{R}[\sigma]^{p \times m}$ we denote the sets of $p \times m$ rational, proper rational and polynomial matrices respectively with real coefficients and indeterminate $\sigma$. A square polynomial matrix

$A(\sigma)=A_{q} \sigma^{q}+A_{q-1} \sigma^{q-1}+\ldots+A_{0} \in \mathbb{R}[\sigma]^{r \times r}, A_{j} \in \mathbb{R}^{r \times r}, j=0,1, \ldots, q \geq 1, A_{q} \neq 0$

is nonsingular iff $\operatorname{det} A(\sigma) \neq 0$ for almost every $\sigma \in \mathbb{C}$. The (finite) zeros of $A(\sigma)$ are defined as the roots of the equation $\operatorname{det} A(\sigma)=0$, equivalently $\lambda_{i} \in \mathbb{C}$ is a (finite) zero of $A(\sigma)$ iff $\operatorname{rank}_{\mathbb{C}} A\left(\lambda_{i}\right)<r$. Assume that $A(\sigma)$ has $l$ distinct zeros $\lambda_{1}, \lambda_{2}, \ldots, \lambda_{l} \in \mathbb{C}$, and let

$$
S_{A(\sigma)}^{\lambda_{i}}=\operatorname{diag}\left\{\left(\sigma-\lambda_{i}\right)^{m_{i 1}}, \ldots,\left(\sigma-\lambda_{i}\right)^{m_{i r}}\right\}
$$

be the local Smith form of $A(\sigma)$ at $\sigma=\lambda_{i}, i=1,2, \ldots, l$ where $m_{i j} \in \mathbb{Z}^{+}$and $0 \leq$ $m_{i 1} \leq m_{i 2} \leq \ldots \leq m_{i r}$. The terms $\left(\sigma-\lambda_{i}\right)^{m_{i j}}$ are called the finite elementary divisors of $A(\sigma)$ at $\sigma=\lambda_{i}$. The non-negative integers $m_{i j}, j=1,2, \ldots, r$ are the partial multiplicities of $\lambda_{i}$ and $m_{i}:=\sum_{j=1}^{r} m_{i j}, i=1,2, \ldots, l$ is the multiplicity

\footnotetext{
${ }^{2}$ rather than the set $\mathbb{Z}^{+}$of nonnegative integers
} 
of $\lambda_{i}$. Let

$$
J_{i j}:=\left[\begin{array}{cccccc}
\lambda_{i} & 1 & 0 & \ldots & 0 & 0 \\
0 & \lambda_{i} & 1 & \ldots & 0 & 0 \\
\vdots & \vdots & \vdots & \ddots & \vdots & \vdots \\
0 & 0 & 0 & \ldots & \lambda_{i} & 1 \\
0 & 0 & 0 & \ldots & 0 & \lambda_{i}
\end{array}\right] \in \mathbb{R}^{m_{i j} \times m_{i j}}, \begin{aligned}
& i=1,2, \ldots, l \\
& j=1,2, \ldots, r
\end{aligned}
$$

be the Jordan block corresponding to the finite elementary divisors $\left(\sigma-\lambda_{i}\right)^{m_{i j}}$ of $A(\sigma)$ and

$$
J_{i}:=\text { block diag }\left[\begin{array}{llll}
J_{i 1}, & J_{i, 2}, & \ldots & , J_{i r}
\end{array}\right] \in \mathbb{R}^{m_{i} \times m_{i}}, \quad i=1,2, \ldots, l
$$

A pair of matrices $C_{i} \in \mathbb{R}^{r \times m_{i}}, J_{i} \in \mathbb{R}^{m_{i} \times m_{i}}$ is called a (finite) Jordan pair of $A(\sigma)[10]$ corresponding to the zero of $A(\sigma)$ at $\sigma=\lambda_{i}$ iff

$$
A_{q} C_{i} J_{i}^{q}+A_{q-1} C_{i} J_{i}^{q-1}+\ldots+A_{1} C_{i} J_{i}+A_{0} C_{i}=0_{r, m_{i}}
$$

and

$$
\operatorname{rank}_{\mathbb{R}}\left[\begin{array}{c}
C_{i} \\
C_{i} J_{i} \\
\vdots \\
C_{i} J_{i}^{m_{i}-1}
\end{array}\right]=m_{i}
$$

Let $n:=\operatorname{deg}(\operatorname{det} A(\sigma))=\sum_{i=1}^{l} m_{i}$. The pair of matrices

$$
\begin{aligned}
C_{F}: & =\left[C_{1}, C_{2}, \ldots, C_{l}\right] \in \mathbb{R}^{r \times n} \\
J_{F} & :=\operatorname{diag}\left\{J_{1}, J_{2}, \ldots, J_{l}\right\} \in \mathbb{R}^{n \times n}
\end{aligned}
$$

is defined as a finite spectral pair [10] of $A(\sigma)$ and by consequence of (13) and (14) satisfies the following conditions

$$
\begin{gathered}
A_{q} C_{F} J_{F}^{q}+A_{q-1} C_{F} J_{F}^{q-1}+\ldots+A_{1} C_{F} J_{F}+A_{0} C_{F}=0_{r, n} \\
\operatorname{rank}_{\mathbb{R}}\left[\begin{array}{c}
C_{F} \\
C_{F} J_{F} \\
\vdots \\
C_{F} J_{F}^{n-1}
\end{array}\right]=n
\end{gathered}
$$

If $A_{0} \neq 0$, the dual matrix $\tilde{A}(\sigma)$ of $A(\sigma)$ is defined as $\tilde{A}(\sigma):=\sigma^{q} A\left(\sigma^{-1}\right)=$ $A_{0} \sigma^{q}+A_{1} \sigma^{q-1}+\ldots+A_{q}$. Since $\operatorname{rank} \tilde{A}(0)=\operatorname{rank} A_{q}$ the dual matrix $\tilde{A}(\sigma)$ of $A(\sigma)$ has zeros at $\sigma=0$ iff $\operatorname{rank} A_{q}<r$. Let $\operatorname{rank} A_{q}<r$ and let

$$
S_{\widetilde{A}(\sigma)}^{0}=\operatorname{diag}\left\{\sigma^{\mu_{1}}, \ldots, \sigma^{\mu_{r}}\right\}
$$

be the local Smith form of $\tilde{A}(\sigma)$ at $\sigma=0$ where $\mu_{j} \in \mathbb{Z}^{+}$and $0 \leq \mu_{1} \leq \mu_{2} \leq \ldots \leq$ $\mu_{r}$. The infinite elementary divisors of $A(\sigma)$ are defined as the finite elementary divisors $\sigma^{\mu_{j}}$ of its dual $\tilde{A}(\sigma)$ at $\sigma=0$. Let

$$
J_{j \infty}:=\left[\begin{array}{cccccc}
0 & 1 & 0 & \ldots & 0 & 0 \\
0 & 0 & 1 & \ldots & 0 & 0 \\
\vdots & \vdots & \vdots & \ddots & \vdots & \vdots \\
0 & 0 & 0 & \ldots & 0 & 1 \\
0 & 0 & 0 & \ldots & 0 & 0
\end{array}\right] \in \mathbb{R}^{\mu_{j} \times \mu_{j}}, j=1,2, \ldots, r
$$


be the Jordan block corresponding to the non-trivial finite elementary divisors $\sigma^{\mu_{j}}, \mu_{j}>0$ of $\tilde{A}(\sigma), \mu:=\sum_{j=1}^{r} \mu_{j}$ and

$$
J_{\infty}:=\operatorname{block} \operatorname{diag}\left[\begin{array}{llll}
J_{1 \infty}, & J_{2 \infty}, & \cdots & , J_{r \infty}
\end{array}\right] \in \mathbb{R}^{\mu \times \mu}
$$

A finite Jordan pair $C_{\infty} \in \mathbb{R}^{r \times \mu}, J_{\infty} \in \mathbb{R}^{\mu \times \mu}$ of the dual matrix $\tilde{A}(\sigma)$ corresponding to the zero of $\tilde{A}(\sigma)$ at $\sigma=0$ is defined as an infinite spectral pair of $A(\sigma)$ and according to (17) and (18) satisfies the following conditions

$$
\begin{gathered}
A_{0} C_{\infty} J_{\infty}^{q}+A_{1} C_{\infty} J_{\infty}^{q-1}+\ldots+A_{q} C_{\infty}=0_{r, \mu} \\
\operatorname{rank}_{\mathbb{R}}\left[\begin{array}{c}
C_{\infty} \\
C_{\infty} J_{\infty} \\
\vdots \\
C_{\infty} J_{\infty}^{\mu-1}
\end{array}\right]=\mu
\end{gathered}
$$

Let

$$
S_{A(\sigma)}^{\infty}(\sigma)=\operatorname{diag}[\underbrace{\sigma^{q_{1}}, \ldots, \sigma^{q_{k}}, I_{v-k}}_{v}, \underbrace{\frac{1}{\sigma^{\widehat{q}_{v+1}}}, \ldots, \frac{1}{\sigma^{\widehat{q}_{r}}}}_{r-v}]
$$

be the Smith-McMillan form of $A(\sigma)$ at $\sigma=\infty[13]$ where

$$
\begin{gathered}
q_{1} \geq q_{2} \geq \ldots \geq q_{k}>0=q_{k+1}=\ldots=q_{v} \\
\widehat{q}_{r} \geq \widehat{q}_{r-1} \geq \ldots \geq \widehat{q}_{v+1}>0
\end{gathered}
$$

are respectively the orders of the poles and the zeros at $\sigma=\infty$ of $A(\sigma)$. Then it is proved in [13] that

$$
q_{1}=q
$$

and that the local Smith form $S_{\tilde{A}(\sigma)}^{0}(\sigma)$ of $\tilde{A}(\sigma)$ at $\sigma=0$ is given by

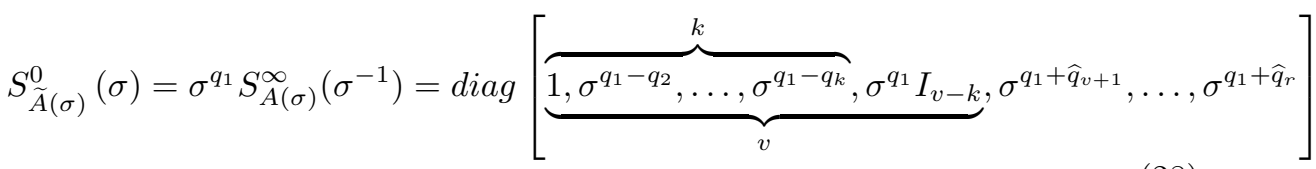

so that the orders $\mu_{j}$ of the infinite elementary divisors $\sigma^{\mu_{j}}, j=1,2, \ldots, r$ of $A(\sigma)$ are given by

$$
\begin{gathered}
\mu_{1}=0 \\
\mu_{j}=q_{1}-q_{j} \quad j=2,3, \ldots, k \\
\mu_{j}=q_{1} \quad j=k+1, \ldots, v \\
\mu_{j}=q_{1}+\widehat{q}_{j} \quad j=v+1, \ldots, r
\end{gathered}
$$

and thus we have

Proposition 1 The total number of elementary divisors (finite and infinite) of $A(\sigma)$ is equal to the product $r q$, where $r$ is the dimension and $q$ is the degree of $A(\sigma)$, i.e.

$$
n+\mu=r q
$$


Proof. For any nonsingular polynomial matrix $A(\sigma)$ we have that [13] total number of zeros of $A(\sigma)$ in $\mathbb{C} \cup\{\infty\}=$ total number of poles of $A(\sigma)$ in $\mathbb{C} \cup\{\infty\}$

or

$$
n+\sum_{j=v+1}^{r} \widehat{q}_{j}=\sum_{j=1}^{k} q_{j}
$$

so that

$$
\begin{aligned}
n+\mu= & n+\sum_{j=1}^{r} \mu_{j} \\
= & n+\underbrace{\left(q_{1}-q_{1}\right)+\left(q_{1}-q_{2}\right)+\ldots+\left(q_{1}-q_{k}\right)}_{k \text { terms }}+(v-k) q_{1} \\
& +\underbrace{\left(q_{1}+\widehat{q}_{v+1}\right)+\ldots+\left(q_{1}+\widehat{q}_{r}\right)}_{(r-v) \text { terms }} \\
= & n+k q_{1}-\Sigma_{j=1}^{k} q_{j}+(v-k) q_{1}+(r-v) q_{1}+\Sigma_{j=v+1}^{r} \widehat{q}_{j} \\
= & n+k q_{1}-\Sigma_{j=1}^{k} q_{j}+v q_{1}-k q_{1}+r q_{1}-v q_{1}+\Sigma_{j=v+1}^{r} \widehat{q}_{j} \\
= & n-\left(q_{1}+q_{2}+\ldots+q_{k}\right)+r q_{1}+\Sigma_{j=v+1}^{r} \widehat{q}_{j} \\
& \stackrel{(35) \mathcal{E}^{(}(27)}{=} r q
\end{aligned}
$$

Consider now the discrete time $q-t h$ order AR equation

$$
A_{q} \xi_{k+q}+A_{q-1} \xi_{k+q-1}+\ldots+A_{0} \xi_{k}=0, \quad k=0,1,2, \ldots, N-q \geq 0
$$

where $1 \leq q \leq N \in \mathbb{Z}^{+}$, or equivalently

$$
A(\sigma) \xi_{k}=0, k=0,1,2, \ldots, N-q \geq 0
$$

where $A(\sigma)=A_{q} \sigma^{q}+A_{q-1} \sigma^{q-1}+\ldots+A_{0} \in \mathbb{R}[\sigma]^{r \times r}$ is a nonsingular polynomial matrix, $\xi_{k} \in \mathbb{R}^{r}, k=0,1,2, \ldots, N$ is a vector sequence and $\sigma$ denotes the forward shift operator $\sigma^{i} \xi_{k}=\xi_{k+i}$. Notice that as the matrix $A_{q} \in \mathbb{R}^{r \times r}$ is not in general invertible (36) can not be solved by iterating forward, i.e. given the initial conditions $\xi_{0}, \xi_{1}, \ldots, \xi_{q-1}$ determine successively $\xi_{q}, \xi_{q+1}, \ldots, \xi_{N}$. This naturally leads to the restriction of the time domain for $\xi_{k}$ to a finite interval $N$ instead of $\mathbb{Z}^{+}$so that we are interested in the behavior (see bellow) of (37) over a specified time interval $k=0,1,2, \ldots, N \geq q$ and not over $\mathbb{Z}^{+}$.

The results of the present paper should be compared to [1], [2], [3], [4], [5], [8] where similar problems for systems in descriptor form, are treated in a similar manner.

The solution space or behavior $\mathcal{B}_{A(\sigma)}$ of the AR-representation (37) is defined as

$$
\mathcal{B}_{A(\sigma)}=\left\{\xi_{k} \mid \xi_{k} \in \mathbb{R}^{r}, \xi_{k} \text { satisfies (37) for } k=0,1,2, \ldots, N \geq q\right\}
$$

and we have

Theorem 2 [7][17] The behavior of the AR-representation (37) over the finite time interval $k=0,1,2, \ldots, N \geq q$ is given by

$$
\mathcal{B}_{A(\sigma)}=\left\{\xi_{k} \in \mathbb{R}^{r} \mid \xi_{k}=\left[\begin{array}{ll}
C_{F} & C_{\infty}
\end{array}\right] \operatorname{diag}\left[J_{F}^{k}, J_{\infty}^{N-k}\right]\left[\begin{array}{l}
a \\
b
\end{array}\right], a \in \mathbb{R}^{n}, b \in \mathbb{R}^{\mu},\right.
$$




$$
k=0,1,2, \ldots, N \geq q\}
$$

and

$$
\operatorname{dim} \mathcal{B}_{A(\sigma)}=r q=n+\mu
$$

Remark 1 The behavior $\mathcal{B}_{A(\sigma)}$ of the AR-representation (37) is thus proved to be a linear vector space of dimension equal to the product of the dimension $r$ of the polynomial matrix $A(\sigma)$ and the highest degree $q$ of $\sigma$ occurring in it or, from Proposition 1, equal to the total number $n+\mu$ of elementary divisors (finite and infinite ones) of $A(\sigma)$. Notice also that the behavior $\mathcal{B}_{A(\sigma)}$ of (37) depends on the finite and infinite spectral pairs $\left(C_{F} \in \mathbb{R}^{r \times n}, J_{F} \in \mathbb{R}^{n \times n}\right)$ and $\left(C_{\infty} \in \mathbb{R}^{r \times \mu}, J_{\infty} \in \mathbb{R}^{\mu \times \mu}\right)$ respectively of $A(\sigma)$ and thus from (10),(11) and (19),(20) on the (non trivial) finite and infinite elementary divisors $\left(\sigma-\lambda_{i}\right)^{m_{i j}}$ and $\sigma^{\mu_{j}}, i=1,2, \ldots, l, j=1,2, \ldots, r$ of the polynomial matrix $A(\sigma)$.

Theorem 3 [7][17] Given the initial and the final conditions vectors

$$
\begin{aligned}
x_{0}: & =\left[\begin{array}{lllll}
\xi_{0}^{\top} & \xi_{1}^{\top} & \cdots & \xi_{q-2}^{\top} & \xi_{q-1}^{\top}
\end{array}\right]^{\top} \in \mathbb{R}^{r q} \\
x_{N-(q-1)}: & =\left[\begin{array}{lllll}
\xi_{N-(q-1)}^{\top} & \xi_{N-(q-2)}^{\top} & \cdots & \xi_{N-1}^{\top} & \xi_{N}^{\top}
\end{array}\right]^{\top} \in \mathbb{R}^{r q}
\end{aligned}
$$

then (37) has the unique solution

$\xi_{k}=\left[\begin{array}{ll}C_{F} & C_{\infty}\end{array}\right]\left[\begin{array}{cc}J_{F}^{k} & 0 \\ 0 & J_{\infty}^{N-k}\end{array}\right]\left[\begin{array}{cc}M_{F} & 0 \\ 0 & M_{\infty}\end{array}\right]\left[\begin{array}{c}x_{0} \\ x_{N-(q-1)}\end{array}\right], k=0,1,2, \ldots, N \geq q$

iff $x_{0}$ and $x_{N-(q-1)}$ satisfy the compatibility boundary conditions

$$
\left[\begin{array}{c}
x_{0} \\
x_{N-(q-1)}
\end{array}\right] \in \operatorname{ker}\left[\begin{array}{cc}
J_{F}^{N-(q-1)} M_{F} & -M_{F} \\
-M_{\infty} & J_{\infty}^{N-(q-1)} M_{\infty}
\end{array}\right]
$$

where $M_{F} \in \mathbb{R}^{n \times r q}, M_{\infty} \in \mathbb{R}^{\mu \times r q}$ are defined by

$$
\left[\begin{array}{l}
M_{F} \\
M_{\infty}
\end{array}\right]:=\left[\begin{array}{ll}
C_{F} & C_{\infty} J_{\infty}^{q-1} \\
C_{F} J_{F} & C_{\infty} J_{\infty}^{q-2} \\
\vdots & \vdots \\
C_{F} J_{F}^{q-1} & C_{\infty}
\end{array}\right]^{-1} \in \mathbb{R}^{r q \times r q}
$$

(the fact that the matrix inside the brackets in the right hand side of (44) is non-singular is proved in [10]).

The physical interpretation of the above theorem is that the system described by (37) can not in general be considered as causal and it is causal iff $\operatorname{rank} A_{q}=r^{3}$. This is also clear from the fact that, in order to determine uniquely a solution we need to choose both the initial and the final conditions,

\footnotetext{
${ }^{3}$ In such a case $A(\sigma)$ is both row and column proper and its Smith-McMillan form at $s=\infty$ is given by $S_{A(\sigma)}^{\infty}=\sigma^{q} I_{r}$, i.e. $A(\sigma)$ has no zeros at $\sigma=\infty$, having only $r$ poles at $\sigma=\infty$ and the orders of its $r$ poles at $\sigma=\infty$ are $q_{1}=q_{2}=\ldots=q_{r}=q$ so that the orders $\mu_{i}$ of its infinite elementary divisors $\sigma^{\mu_{i}}$ satisfy $\mu_{i}=0, i=1,2, \ldots, r$ so that $\mu=0$ and $r q=n$. See also Corollary 2.5 bellow.
} 
which have to satisfy the compatibility boundary conditions (43). So this is a boundary value problem. These compatibility boundary conditions, have a very clear physical meaning, i.e. they reflect the propagation of the initial conditions in the forward time direction, to the final conditions, and vice versa. This is clear from the decomposition of the solution space $\mathcal{B}_{A(\sigma)}$ into two subspaces the one corresponding to the finite elementary divisors of $A(\sigma)$ and the other corresponding to the infinite elementary divisors. The first part of the solution spanned by $C_{F} J_{F}^{k}$ which depends on the finite elementary divisors gives rise to solutions moving in the forward direction of time and reflects the forward propagation of the initial conditions $\xi_{0}, \xi_{1}, \ldots, \xi_{q-1}$. The second part $C_{\infty} J_{\infty}^{N-k}$ which depends on the infinite elementary divisors gives solutions moving backwards in time, i.e. from the final time $N$ to 0 and reflects the backward propagation of the final conditions $\xi_{N-(q-1)}, \xi_{N-(q-2)}, \ldots, \xi_{N-1}, \xi_{N}$. This discussion should be compared to that in [1][2]. Notice that the above decomposition of the solution space into forward and backward subspaces, corresponds to a maximal forward decomposition of a descriptor system in [3]. Notice also that the variable $k=0,1, \ldots, N$ in (36) rather than representing discrete time instants it may be considered to represent discrete spatial positions within a system. In such a case and if $\operatorname{rank} A_{q}<r$ the non-causality of the discrete time AR representation is replaced by a spacial two boundary value problem specified by the initial and final conditions $\xi_{0}, \xi_{1}, \ldots, \xi_{q-1}$ and $\xi_{N-(q-1)}, \ldots, \xi_{N-1}, \xi_{N}$ governing the spacial steady state behavior of the system and the above analysis shows the importance of the infinite elementary divisors of the polynomial matrix $A(\sigma)$ on the spacial behavior of such systems.

\subsection{Elementary divisors of a matrix pencil linearization of a nonsingular polynomial matrix}

We examine now the structure of the finite and infinite elementary divisors of the matrix pencil in (7) or of what we call a block companion linearization of a nonsingular polynomial matrix $A(\sigma)$. We start with a refined statement of the results contained in formulas $(24)-(32)$.

Lemma 4 Let $A(\sigma)=A_{q} \sigma^{q}+A_{q-1} \sigma^{q-1}+\ldots+A_{1} \sigma+A_{0} \in \mathbb{R}[\sigma]^{r \times r}$ be nonsingular and let the Smith-McMillan form $S_{A(\sigma)}^{\infty}(\sigma)$ of $A(\sigma)$ at $\sigma=\infty$ be given by

$$
S_{A(\sigma)}^{\infty}(\sigma)=\operatorname{diag}[\underbrace{\sigma^{q_{1}} I_{\eta}, \sigma^{q_{\eta+1}}, \ldots, \sigma^{q_{k}}, I_{v-k}}_{v}, \frac{1}{\sigma^{\widehat{q}_{v+1}}}, \ldots, \frac{1}{\sigma^{\widehat{q}_{r}}}]
$$

i.e. assume that for some $\eta \in \mathbb{Z}^{+}: 1 \leq \eta \leq r, q=q_{1}=q_{2}=\ldots=q_{\eta}>q_{\eta+1} \geq$ 0. Then $\eta=\operatorname{rank} A_{q}$.

Proof. Consider the matrix pencil $\sigma \bar{E}-\bar{A} \in \mathbb{R}[s]^{q r \times q r}$ where

$\bar{E}:=\left[\begin{array}{ccccc}I_{r} & 0 & \ldots & 0 & 0 \\ 0 & I_{r} & \ldots & 0 & 0 \\ \vdots & \vdots & \ddots & \vdots & \vdots \\ 0 & 0 & \ldots & I_{r} & 0 \\ 0 & 0 & \ldots & 0 & A_{q}\end{array}\right] \in \mathbb{R}^{q r \times q r}, \bar{A}:=\left[\begin{array}{ccccc}0 & I_{r} & 0 & \ldots & 0 \\ 0 & 0 & I_{r} & \ldots & 0 \\ \vdots & \vdots & \vdots & \ddots & \vdots \\ 0 & 0 & \ldots & 0 & I_{r} \\ -A_{0} & -A_{1} & \ldots & -A_{q-2} & -A_{q-1}\end{array}\right] \in \mathbb{R}^{q r \times q r}$ 
and the biproper rational matrices:

$$
\begin{aligned}
U_{\infty}(\sigma) & :=\left[\begin{array}{ccccc}
I_{r} & 0 & \cdots & 0 & 0 \\
0 & I_{r} & \cdots & 0 & 0 \\
\vdots & \vdots & \vdots & \ddots & \vdots \\
0 & 0 & \cdots & I_{r} & 0 \\
-W_{0}(\sigma) & -W_{1}(\sigma) & \cdots & -W_{q-2}(\sigma) & I_{r}
\end{array}\right] \in \mathbb{R}_{p r}(\sigma)^{q r \times q r}(47) \\
V_{\infty}(\sigma):= & {\left[\begin{array}{cccccc}
I_{r} & I_{r} \sigma^{-1} & I_{r} \sigma^{-2} & \ldots & I_{r} \sigma^{-(q-2)} & I_{r} \sigma^{-(q-1)} \\
0 & I_{r} & I_{r} \sigma^{-1} & \ldots & I_{r} \sigma^{-(q-3)} & I_{r} \sigma^{-(q-2)} \\
\vdots & \vdots & \ldots & \cdots & \vdots & \vdots \\
0 & 0 & 0 & \vdots & I_{r} & I_{r} \sigma^{-1} \\
0 & 0 & 0 & \cdots & 0 & I_{r}
\end{array}\right] \in \mathbb{R}_{p r}(\sigma)^{q r \times q r} }
\end{aligned}
$$

where $W_{i}(\sigma)=\sigma^{-(i+1)} \sum_{k=0}^{i} A_{k} \sigma^{k} \in \mathbb{R}_{p r}(\sigma)^{r \times r}, i=0,1,2, \ldots q-2$. Then, using the fact that $q=q_{1}$, it is easy to verify that

$$
U_{\infty}(\sigma)(\sigma \bar{E}-\bar{A}) V_{\infty}(\sigma)=\operatorname{diag}\left\{\sigma I_{r\left(q_{1}-1\right)}, \frac{1}{\sigma^{q_{1}-1}} A(\sigma)\right\}
$$

and since $U_{\infty}(\sigma)$ and $V_{\infty}(\sigma)$ are biproper rational matrices, the pencil $\sigma \bar{E}-\bar{A}$ and the block diagonal matrix in the right-hand side of (49), are equivalent at $\sigma=\infty[13]$, hence they have identical pole-zero structures at $\sigma=\infty$.

Assume now that the biproper rational matrices $T_{L}(\sigma) \in \mathbb{R}_{p r}(\sigma)^{r \times r}, T_{R}(\sigma) \in$ $\mathbb{R}_{p r}(\sigma)^{r \times r}$ bring the matrix $A(\sigma)$ to its Smith-McMillan form $S_{A(\sigma)}^{\infty}(\sigma)$ at $\sigma=$ $\infty$, i.e. let that

$T_{L}(\sigma) A(\sigma) T_{R}(\sigma)=S_{A(\sigma)}^{\infty}(\sigma)=\operatorname{diag}[\underbrace{\sigma^{q_{1}} I_{\eta}, \sigma^{q_{\eta+1}}, \ldots, \sigma^{q_{k}}, I_{v-k}}_{v}, \frac{1}{\sigma^{\widehat{q}_{v+1}}}, \ldots, \frac{1}{\sigma^{\widehat{q}_{r}}}]$

By pre and post-multiplying (49), respectively by $\hat{T}_{L}(\sigma):=\operatorname{diag}\left[I_{r\left(q_{1}-1\right)}, T_{L}(\sigma)\right] \in$ $\mathbb{R}_{p r}(\sigma)^{q r \times q r}$ and $\hat{T}_{R}(\sigma):=\operatorname{diag}\left[I_{r\left(q_{1}-1\right)}, T_{R}(\sigma)\right] \in \mathbb{R}_{p r}(\sigma)^{q r \times q r}$ we have:

$$
\hat{T}_{L}(\sigma) U_{\infty}(\sigma)(\sigma \bar{E}-\bar{A}) V_{\infty}(\sigma) \hat{T}_{R}(\sigma)=\operatorname{diag}\left[\sigma I_{r\left(q_{1}-1\right)}, \frac{1}{\sigma^{q_{1}-1}} S_{A(\sigma)}^{\infty}(\sigma)\right]
$$

It is obvious that equation (51) gives the Smith-McMillan form $S_{\sigma \bar{E}-\bar{A}}^{\infty}(\sigma)$ of the pencil $\sigma \bar{E}-\bar{A}$ at $\sigma=\infty$, i.e.

$$
\begin{aligned}
& S_{\sigma \bar{E}-\bar{A}}^{\infty}(\sigma) \\
= & \operatorname{diag}\left[\sigma I_{r\left(q_{1}-1\right)}, \frac{1}{\sigma^{q_{1}-1}} S_{A(\sigma)}^{\infty}(\sigma)\right] \\
= & \operatorname{diag}\left[\sigma I_{r\left(q_{1}-1\right)}, \frac{1}{\sigma^{q_{1}-1}} \operatorname{diag}[\underbrace{\sigma^{q_{1}} I_{\eta}, \sigma^{q_{\eta+1}}, \ldots, \sigma^{q_{k}}, I_{v-k}}_{v}, \frac{1}{\sigma^{\widehat{q}_{v+1}}}, \ldots, \frac{1}{\sigma^{\widehat{q}_{r}}}]\right] \\
= & \operatorname{diag}\left[\sigma I_{r\left(q_{1}-1\right)}, \sigma I_{\eta}, \frac{1}{\sigma^{q_{1}-q_{\eta+1}-1}}, \ldots \frac{1}{\sigma^{q_{1}-q_{k}-1}}, \frac{1}{\sigma^{q_{1}-1}} I_{v-k}, \frac{1}{\sigma^{\hat{q}_{v+1}+q_{1}-1}}, \ldots, \frac{1}{\sigma^{\hat{q}_{r}+q_{1}-1}}\right]
\end{aligned}
$$


According to [14] the rank of the matrix $\bar{E}$ is equal to the number of the poles of $\sigma \bar{E}-\bar{A}$ at $\sigma=\infty$ of order equal to 1 , i.e.:

$$
\operatorname{rank} \bar{E}=r\left(q_{1}-1\right)+\eta
$$

But from the construction of $\bar{E}$ it is easy to see that

$$
\operatorname{rank} \bar{E}=r\left(q_{1}-1\right)+\operatorname{rank} A_{q} .
$$

¿From (53) and (54) we have that $\operatorname{rank} A_{q}=\eta$, where, according to (45), $\eta$ is the number of poles of $A(\sigma)$ at $\sigma=\infty$ of order $q=q_{1}$.

Corollary 5 If $r>\operatorname{rank} A_{q}=\eta \geq 1$ so that $q=q_{1}=q_{2}=\ldots=q_{\eta}>q_{\eta+1}$, then the orders $\mu_{j}$ of the infinite elementary divisors $\sigma^{\mu_{j}}$ of $A(\sigma)$ are given by

$$
\begin{aligned}
& \mu_{j}=0, \quad j=1,2, \ldots, \eta \\
& \mu_{j}=q_{1}-q_{j} \quad j=\eta+1, \eta+2, \ldots, k \\
& \mu_{j}=q_{1} \quad j=k+1, \ldots, v \\
& \mu_{j}=q_{1}+\widehat{q}_{j} \quad j=v+1, \ldots, r
\end{aligned}
$$

Proof. This follows from the fact that if $q=q_{1}=q_{2}=\ldots=q_{\eta}>q_{\eta+1}$, then $S_{A(\sigma)}^{\infty}(\sigma)$ is given by (45) and thus the local Smith form $S_{\widetilde{A}(\sigma)}^{0}(\sigma)$ of $\tilde{A}(\sigma)$ at $\sigma=0$ is given by

$$
\begin{aligned}
S_{\widetilde{A}(\sigma)}^{0}(\sigma) & =\sigma^{q_{1}} S_{A(\sigma)}^{\infty}\left(\sigma^{-1}\right) \\
& =\sigma^{q_{1}} \operatorname{diag}[\underbrace{\sigma^{-q_{1}} I_{\eta}, \sigma^{-q_{\eta+1}}, \ldots, \sigma^{-q_{k}}, I_{v-k}}_{v}, \sigma^{\widehat{q}_{v+1}}, \ldots, \sigma^{\widehat{q}_{r}}] \\
& =\operatorname{diag}[\underbrace{I_{\eta}, \sigma^{q_{1}-q_{\eta+1}}, \ldots, \sigma^{q_{1}-q_{k}}, \sigma^{q_{1}} I_{v-k}}_{v}, \sigma^{q_{1}+\widehat{q}_{v+1}}, \ldots, \sigma^{q_{1}+\widehat{q}_{r}}]
\end{aligned}
$$

Lemma 6 Let $A(\sigma)=A_{q} \sigma^{q}+A_{q-1} \sigma^{q-1}+\ldots+A_{1} \sigma+A_{0} \in \mathbb{R}[\sigma]^{r \times r}$ a nonsingular polynomial matrix. Then the matrix pencil $\sigma \bar{E}-\bar{A} \in \mathbb{R}[\sigma]^{q r \times q r}$ in (7) and $A(\sigma)$, have isomorphic finite elementary divisors structure and thus the same finite zero structure and isomorphic non-trivial infinite elementary divisor structure.

Proof. First we shall prove that the pencil $\sigma \bar{E}-\bar{A}$ and the polynomial matrix $A(\sigma)$, have the same non-trivial infinite elementary divisors.

The infinite elementary divisors of $\sigma \bar{E}-\bar{A}$ are the finite elementary divisors $\sigma^{\bar{\mu}_{j}}$ of the dual pencil $\sigma\left(\sigma^{-1} \bar{E}-\bar{A}\right)=\bar{E}-\sigma \bar{A}$ at $\sigma=0$, and according to the formula (28) the local Smith form $S_{\bar{E}-\sigma \bar{A}}^{0}(\sigma)$ at $\sigma=0$ of the dual pencil $\bar{E}-\sigma \bar{A}$ is given by

$$
\begin{aligned}
S_{\bar{E}-\sigma \bar{A}}^{0}(\sigma)= & \sigma S_{\sigma \bar{E}-\bar{A}}^{\infty}\left(\sigma^{-1}\right) \\
& \stackrel{(52)}{=} \sigma \operatorname{diag}\left[\frac{1}{\sigma} I_{r\left(q_{1}-1\right)}, \sigma^{q_{1}-1} S_{A(\sigma)}^{\infty}\left(\frac{1}{\sigma}\right)\right]
\end{aligned}
$$




$$
\begin{aligned}
= & \operatorname{diag}\left[I_{r\left(q_{1}-1\right)}, \sigma^{q_{1}} S_{A(\sigma)}^{\infty}\left(\frac{1}{\sigma}\right)\right] \\
& \stackrel{(50)}{=} \operatorname{diag}[I_{r\left(q_{1}-1\right)}, \overbrace{\underbrace{r}_{I_{\eta}, \sigma^{q_{1}-q_{\eta+1}}, \ldots, \sigma^{q_{1}-q_{k}}, \sigma^{q_{1}} I_{v-k}}, \sigma^{q_{1}+\widehat{q}_{v+1}}, \ldots, \sigma^{q_{1}+\widehat{q}_{r}}}^{r}]
\end{aligned}
$$

so that the orders $\bar{\mu}_{j}$ of the infinite elementary divisors $\sigma^{\bar{\mu}_{j}}$ of $\sigma \bar{E}-\bar{A}$ are given by

$$
\begin{aligned}
& \bar{\mu}_{j}=0=\mu_{j}, \quad j=1,2, \ldots, r\left(q_{1}-1\right)+\eta \\
& \bar{\mu}_{j}=q_{1}-q_{j}=\mu_{j} \quad j=\eta+1, \eta+2, \ldots, k \\
& \bar{\mu}_{j}=q_{1}=\mu_{j} \quad j=k+1, \ldots, v \\
& \bar{\mu}_{j}=q_{1}+\widehat{q}_{j}=\mu_{j} \quad j=v+1, \ldots, r
\end{aligned}
$$

¿From (55) in Corollary 5 and (58) we see that the polynomial matrix $A(\sigma) \in$ $\mathbb{R}[\sigma]^{r \times r}$ and the matrix pencil $\sigma \bar{E}-\bar{A} \in \mathbb{R}[\sigma]^{q r \times q r}$ have identical non-trivial infinite elementary divisor structures.

We now prove that the pencil $\sigma \bar{E}-\bar{A}$ and the polynomial matrix $A(\sigma)$ have identical finite elementary divisors and thus the same finite zero structures. Consider the $\mathbb{R}[\sigma]$-unimodular matrices:

$$
\begin{gathered}
U(\sigma)=\left[\begin{array}{ccccc}
-I_{r} & 0 & \ldots & 0 & 0 \\
0 & -I_{r} & \ldots & 0 & 0 \\
\vdots & \vdots & \vdots & \vdots & \vdots \\
0 & 0 & 0 & -I_{r} & 0 \\
T_{q-1}(\sigma) & T_{q-2}(\sigma) & \ldots & T_{1}(\sigma) & I_{r}
\end{array}\right] \in \mathbb{R}[\sigma]^{q r \times q r} \\
V(\sigma)=\left[\begin{array}{cccccc}
0 & 0 & \ldots & \ldots & 0 & I_{r} \\
I_{r} & 0 & \ldots & \ldots & 0 & \sigma I_{r} \\
\sigma I_{r} & I_{r} & \ldots & \ldots & 0 & \sigma^{2} I_{r} \\
\vdots & \vdots & \ddots & \ldots & \vdots & \vdots \\
\sigma^{q-3} I_{r} & \sigma^{q-4} I_{r} & \ldots & I_{r} & 0 & \sigma^{q-2} I_{r} \\
\sigma^{q-2} I_{r} & \sigma^{q-3} I_{r} & \ldots & \sigma I_{r} & I_{r} & \sigma^{q-1} I_{r}
\end{array}\right] \in \mathbb{R}[\sigma]^{q r \times q r}
\end{gathered}
$$

where $T_{i}(\sigma)=\sigma T_{i-1}(\sigma)+A_{q_{1}-i}, i=1,2, \ldots, q-1, T_{0}=A_{q}$. It is then easy to verify the equation

$$
U(\sigma)(\sigma \bar{E}-\bar{A}) V(\sigma)=\operatorname{diag}\left[I_{r(q-1)}, A(\sigma)\right]
$$

Consider the Smith form $S_{A(\sigma)}^{\mathbb{C}}(\sigma)$ in $\mathbb{C}$ of $A(\sigma)$ and let $\bar{T}_{L}(\sigma) \in \mathbb{R}[\sigma]^{r \times r}, \bar{T}_{R}(\sigma) \in$ $\mathbb{R}[\sigma]^{r \times r}$ and $\mathbb{R}[\sigma]$-unimodular such that

$$
\bar{T}_{L}(\sigma) A(\sigma) \bar{T}_{R}(\sigma)=S_{A(\sigma)}^{\mathbb{C}}(\sigma)
$$

Pre-and post-multiplying $(61)$, respectively by $\tilde{T}_{L}(\sigma):=\operatorname{diag}\left[I_{r(q-1)}, \bar{T}_{L}(\sigma)\right]$ and $\tilde{T}_{R}(\sigma):=\operatorname{diag}\left[I_{r(q-1)}, \bar{T}_{R}(\sigma)\right]$, we have

$$
\tilde{T}_{L}(\sigma) U(\sigma)(\sigma \bar{E}-\bar{A}) V(\sigma) \tilde{T}_{R}(\sigma)=\operatorname{diag}\left[I_{r(q-1)}, S_{A(\sigma)}^{\mathbb{C}}(\sigma)\right]
$$


Equation (63) obviously gives the Smith form $S_{\sigma \bar{E}-\bar{A}}^{\mathbb{C}}(\sigma)$ in $\mathbb{C}$ of the matrix pencil $\sigma \bar{E}-\bar{A}$, i.e.

$$
S_{\sigma \bar{E}-\bar{A}}^{\mathbb{C}}(\sigma)=\operatorname{diag}\left[I_{r\left(q_{1}-1\right)}, S_{A(\sigma)}^{\mathbb{C}}(\sigma)\right]
$$

So the polynomial matrix $A(\sigma) \in \mathbb{R}[\sigma]^{r \times r}$ and the matrix pencil $\sigma \bar{E}-\bar{A} \in$ $\mathbb{R}[\sigma]^{q r \times q r}$ have the same zero structure in $\mathbb{C}$ and thus the same finite elementary divisors.

\section{First order realization of a discrete-time AR- representation}

In this section we deal with the application of the results presented above to what we define as a first order realization of a discrete time AR representation as in (36). By this term we mean an AR-representation having the form (36) with order $q=1$ and such that both AR-representations have isomorphic behaviors. Equivalence of continuous time linear systems has been the subject of several studies in the past. The definition of equivalence in our case is similar to that of fundamental equivalence [18][6] of continuous time systems.

Consider the discrete time AR-representation

$$
A(\sigma) \xi_{k}=0, \quad k=0,1,2, \ldots, N-q \geq 0
$$

with $A(\sigma) \in \mathbb{R}[\sigma]^{r \times r}$ nonsingular as in (9) and its behavior:

$$
\begin{aligned}
\mathcal{B}_{A(\sigma)} & =\left\{\xi_{k} \in \mathbb{R}^{r} \mid \xi_{k}=\left[C_{F}, C_{\infty}\right] \operatorname{diag}\left[J_{F}^{k} M_{F}, J_{\infty}^{N-k} M_{\infty}\right]\left[\begin{array}{c}
x_{0} \\
x_{N-(q-1)}
\end{array}\right],\right. \\
k & =0,1,2, \ldots, N \geq q
\end{aligned}
$$

where the initial and final conditions vectors:

$$
x_{0}:=\left[\begin{array}{lllll}
\xi_{0}^{\top} & \xi_{1}^{\top} & \cdots & \xi_{q-2}^{\top} & \xi_{q-1}^{\top}
\end{array}\right]^{\top} \in \mathbb{R}^{r q}
$$

and

$$
x_{N-(q-1)}:=\left[\begin{array}{lllll}
\xi_{N-(q-1)}^{\top} & \xi_{N-(q-2)}^{\top} & \cdots & \xi_{N-1}^{\top} & \xi_{N}^{\top}
\end{array}\right]^{\top} \in \mathbb{R}^{r q}
$$

satisfy the compatibility boundary condition (43) and define the vector

$$
x_{k}:=\left[\begin{array}{l}
\xi_{k} \\
\xi_{k+1} \\
\vdots \\
\xi_{k+q-2} \\
\xi_{k+q-1}
\end{array}\right] \in \mathbb{R}^{r q}, \quad k=0,1,2, \ldots, N-(q-1)
$$

In view of (65) and the forms of $\bar{E}$ and $\bar{A}$ in (46) the vector $x_{k}$ satisfies the equation

$$
\bar{E} x_{k+1}=\bar{A} x_{k}, \quad k=0,1,2, \ldots, N-q
$$


or equivalently the discrete time AR-representation

$$
(\sigma \bar{E}-\bar{A}) x_{k}=0, \quad k=0,1,2, \ldots, N-q
$$

with behavior

$$
\begin{aligned}
\mathcal{B}_{\sigma \bar{E}-\bar{A}} & =\left\{x_{k} \in \mathbb{R}^{r q} \mid x_{k}=\left[\bar{C}_{F}, \bar{C}_{\infty}\right] \operatorname{diag}\left[\bar{J}_{F}^{k} M_{F}, \bar{J}_{\infty}^{N-k-(q-1)} M_{\infty}\right]\left[\begin{array}{l}
x_{0} \\
x_{N-(q-1)}
\end{array}\right],\right. \\
k & =0,1,2, \ldots, N-(q-1)\}
\end{aligned}
$$

and from Theorem 2

$$
\operatorname{dim} \mathcal{B}_{\sigma \bar{E}-\bar{A}}=(r q) \cdot 1=n+m
$$

where $\bar{C}_{F} \in \mathbb{R}^{r q \times n}, \bar{J}_{F} \equiv J_{F} \in \mathbb{R}^{n \times n}$ a finite Jordan pair and $\bar{C}_{\infty} \in \mathbb{R}^{r q \times \mu}, \bar{J}_{\infty} \equiv$ $J_{\infty} \in \mathbb{R}^{\mu \times \mu}$ an infinite Jordan pair respectively of $[\sigma \bar{E}-\bar{A}] \in \mathbb{R}[\sigma]^{r q \times r q}$, and it can easily be proved that

$$
\left[\bar{C}_{F}, \bar{C}_{\infty}\right]=\left[\begin{array}{ll}
C_{F} & C_{\infty} J_{\infty}^{q-1} \\
C_{F} J_{F} & C_{\infty} J_{\infty}^{q-2} \\
\vdots & \vdots \\
C_{F} J_{F}^{q-1} & C_{\infty}
\end{array}\right]
$$

$M_{F} \in \mathbb{R}^{n \times r q}, M_{\infty} \in \mathbb{R}^{\mu \times r q}$ and defined through

$$
\left[\begin{array}{c}
M_{F} \\
M_{\infty}
\end{array}\right]:=\left[\bar{C}_{F}, \bar{C}_{\infty}\right]^{-1} \in \mathbb{R}^{r q \times r q}
$$

and where the initial and final conditions vectors $x_{0} \in \mathbb{R}^{r q}, x_{N-(q-1)} \in \mathbb{R}^{r q}$ satisfy the compatibility boundary condition:

$$
\left[\begin{array}{l}
x_{0} \\
x_{N-(q-1)}
\end{array}\right] \in \operatorname{ker}\left[\begin{array}{ll}
J_{F}^{N-(q-1)} M_{F} & -M_{F} \\
-M_{\infty} & J_{\infty}^{N-(q-1)} M_{\infty}
\end{array}\right]
$$

Definition 1 Two AR-representations

$$
A_{i}(\sigma) \xi_{k}^{i}=0, \quad k=0,1,2, \ldots, N-q^{i} \geq 0, \quad i=1,2
$$

where $A_{i}(\sigma)=A_{i q^{i}} \sigma^{q^{i}}+A_{i q^{i}-1} \sigma^{q^{i}-1}+\ldots+A_{i 1} \sigma+A_{i 0} \in \mathbb{R}[\sigma]^{r_{i} \times r_{i}}, i=1,2$ will be called fundamentally equivalent over the finite time interval $k=0,1,2, \ldots N$ iff there exists a bijective polynomial map between their respective behaviors $\mathcal{B}_{A_{1}(\sigma)}, \mathcal{B}_{A_{2}(\sigma)}$.

Using the results of the previous section we can prove the following

Theorem 7 The discrete time AR representations in (65) and (68) are fundamentally equivalent over the finite time interval $k=0,1,2, \ldots, N$.

Proof. According to Definition 1 the AR representations (65) and (68) are fundamentally equivalent over the finite time interval $k=0,1,2, \ldots, N$ iff there exist bijective polynomial maps between their respective behaviors:

$$
S_{r q}(\sigma) \in \mathbb{R}[\sigma]^{r q \times r}: \mathcal{B}_{A(\sigma)} \rightarrow \mathcal{B}_{\sigma \bar{E}-\bar{A}} \mid \xi_{k} \mapsto x_{k}=S_{r q}(\sigma) \xi_{k} \in \mathcal{B}_{\sigma \bar{E}-\bar{A}}
$$




$$
L_{r q}(\sigma) \in \mathbb{R}[\sigma]^{r \times r q}: \mathcal{B}_{\sigma \bar{E}-\bar{A}} \rightarrow \mathcal{B}_{A(\sigma)} \mid x_{k} \mapsto \xi_{k}=L_{r q}(\sigma) x_{k} \in \mathcal{B}_{A(\sigma)}
$$

As it can be seen from the definition of $x_{k}$ :

$$
x_{k}:=\left[\begin{array}{l}
\xi_{k} \\
\xi_{k+1} \\
\vdots \\
\xi_{k+q-2} \\
\xi_{k+q-1}
\end{array}\right]=\left[\begin{array}{c}
I_{r} \\
I_{r} \sigma \\
\vdots \\
I_{r} \sigma^{q-2} \\
I_{r} \sigma^{q-1}
\end{array}\right] \xi_{k}, \quad k=0,1,2, \ldots, N-(q-1)
$$

the polynomial maps $S_{r q}(\sigma)$ and $L_{r q}(\sigma)$ required by (70) and (71), are respectively given by

$$
\begin{gathered}
S_{r q}(\sigma)=\left[\begin{array}{c}
I_{r} \\
I_{r} \sigma \\
\vdots \\
I_{r} \sigma^{q-2} \\
I_{r} \sigma^{q-1}
\end{array}\right] \in \mathbb{R}[\sigma]^{r q \times r} \\
L_{r q}(\sigma)=\left[\begin{array}{llll}
I_{r} & 0 & \ldots & 0
\end{array}\right] \in \mathbb{R}^{r \times r q}
\end{gathered}
$$

Moreover and according to the results of the previous section, the polynomial matrices $A(\sigma)$ and $\sigma \bar{E}-\bar{A}$, will have isomorphic finite and infinite elementary divisors structure which implies that

$$
\operatorname{dim} \mathcal{B}_{A(\sigma)}=\operatorname{dim} \mathcal{B}_{\sigma \bar{E}-\bar{A}}=r q=n+\mu
$$

Taking into account the fact that for $\xi_{k} \in \mathcal{B}_{A(\sigma)}$ such that

$$
S_{r q}(\sigma) \xi_{k}=0
$$

then

$$
\xi_{k}=0, \quad k=0,1, \ldots, N
$$

we conclude that $S_{r q}(\sigma)$ is injective. Now

$$
\operatorname{Im} S_{r q}(\sigma):=\left\{x_{k} \mid x_{k}=S_{r q}(\sigma) \xi_{k}, \xi_{k} \in \mathcal{B}_{A(\sigma)}\right\} \subseteq \mathcal{B}_{\sigma \bar{E}-\bar{A}}
$$

Taking into account (75) it follows that

$$
\operatorname{Im} S_{r q}(\sigma)=\mathcal{B}_{\sigma \bar{E}-\bar{A}}
$$

and so $S_{r q}(\sigma)$ is surjective. Thus $S_{r q}(\sigma)$ is the polynomial bijective map required by (70). Similar argument prove that $L(\sigma)$ in $(74)$ is the polynomial bijective map required by (71).

Definition 2 The AR-representation (68) will be called a first order realization of the AR-representation (65) over the finite time interval $k=0,1,2, \ldots, N$. 


\section{Strict equivalence of polynomial matrices and fundamental equivalence of discrete-time AR- representations}

Consider the discrete-time AR-representation

$$
A(\sigma) \xi_{k}=0, \quad k=0,1,2, \ldots, N-q
$$

with $A(\sigma) \in \mathbb{R}[\sigma]^{r \times r}$ as in (9) and its first order realization over the finite time interval $k=0,1,2, \ldots, N$ :

$$
(\sigma \bar{E}-\bar{A}) x_{k}=0, \quad k=0,1,2, \ldots, N-q
$$

where $\bar{E}$ and $\bar{A}$ as in (46). Let now $M \in \mathbb{R}^{r q \times r q}, Q \in \mathbb{R}^{r q \times r q}$ and nonsingular and such that the matrix pencil

$$
[\sigma E-A]:=M[\sigma \bar{E}-\bar{A}] Q \in \mathbb{R}[\sigma]^{r q \times r q}
$$

and $[\sigma \bar{E}-\bar{A}]$ are strictly equivalent [9][14]. We can choose the matrices $M \in$ $\mathbb{R}^{r q \times r q}$ and $Q \in \mathbb{R}^{r q \times r q}$ so that $[\sigma E-A]$ is in the Weierstrass canonical form $[9]$. We state this fact in

Lemma 8 With

$$
Q:=\left[\begin{array}{ll}
C_{F} & C_{\infty} J_{\infty}^{q-1} \\
C_{F} J_{F} & C_{\infty} J_{\infty}^{q-2} \\
\vdots & \vdots \\
C_{F} J_{F}^{q-1} & C_{\infty}
\end{array}\right] \in \mathbb{R}^{r q \times r q}
$$

and

$\left.M:=\left[\begin{array}{lll}C_{F} & \ldots & C_{\infty} J_{\infty}^{q-2} \\ C_{F} J_{F} & \ldots & C_{\infty} J_{\infty}^{q-3} \\ \vdots & \ldots & \vdots \\ C_{F} J_{F}^{q-2} & \ldots & C_{\infty} \\ A_{q} C_{F} J_{F}^{q-1} & \ldots & -\left[A_{0} C_{\infty} J_{\infty}^{q-1}+A_{1} C_{\infty} J_{\infty}^{q-2}+\ldots+A_{q-1} C_{\infty}\right]\end{array}\right]\right]^{-1} \in \mathbb{R}^{r q \times r q}$

the pencil $\sigma E-A$ in (79) is in the Weierstrass canonical form given by

$$
[\sigma E-A]=M[\sigma \bar{E}-\bar{A}] Q=\left[\begin{array}{ll}
\sigma I_{n}-J_{F} & 0_{n, \mu} \\
0_{\mu, n} & \sigma J_{\infty}-I_{\mu}
\end{array}\right]
$$

Proof. Conditions (17) and (22)

$$
\begin{gathered}
A_{q} C_{F} J_{F}^{q}+A_{q-1} C_{F} J_{F}^{q-1}+\ldots+A_{1} C_{F} J_{F}+A_{0} C_{F}=0_{r, m} \\
A_{0} C_{\infty} J_{\infty}^{q}+A_{1} C_{\infty} J_{\infty}^{q-1}+\ldots+A_{q} C_{\infty}=0_{r, \mu}
\end{gathered}
$$

imply respectively that

$$
A_{q-1} C_{F} J_{F}^{q-1}+\ldots+A_{1} C_{F} J_{F}+A_{0} C_{F}=-A_{q} C_{F} J_{F}^{q}
$$




$$
\sigma A_{q} C_{\infty}=-\sigma\left(A_{0} C_{\infty} J_{\infty}^{q}+A_{1} C_{\infty} J_{\infty}^{q-1}+\ldots+A_{q-1} J_{\infty} C_{\infty}\right)
$$

So by direct calculation we have

$$
\begin{aligned}
& {[\sigma \bar{E}-\bar{A}] Q} \\
& =\left[\begin{array}{ccccc}
\sigma I_{r} & -I_{r} & \ldots & 0 & 0 \\
0 & \sigma I_{r} & \ldots & 0 & 0 \\
\vdots & \vdots & \ddots & \vdots & \vdots \\
0 & 0 & \ldots & \sigma I_{r} & -I_{r} \\
A_{0} & A_{1} & \ldots & A_{q-2} & \sigma A_{q}+A_{q-1}
\end{array}\right]\left[\begin{array}{ll}
C_{F} & C_{\infty} J_{\infty}^{q-1} \\
C_{F} J_{F} & C_{\infty} J_{\infty}^{q-2} \\
\vdots & \vdots \\
C_{F} J_{F}^{q-1} & C_{\infty}
\end{array}\right] \\
& =\left[\begin{array}{lll}
C_{F}\left(\sigma I_{n}-J_{F}\right) & \ldots & C_{\infty} J_{\infty}^{q-2}\left(\sigma J_{\infty}-I_{\mu}\right) \\
C_{F} J_{F}\left(\sigma I_{n}-J_{F}\right) & \ldots & C_{\infty} J_{\infty}^{q-3}\left(\sigma J_{\infty}-I_{\mu}\right) \\
\vdots & \ldots & \vdots \\
C_{F} J_{F}^{q-2}\left(\sigma I_{n}-J_{F}\right) & \ldots & C_{\infty}\left(\sigma J_{\infty}-I_{\mu}\right) \\
A_{0} C_{F}+A_{1} C_{F} J_{F}+\ldots & \ldots & A_{0} C_{\infty} J_{\infty}^{q-1}+A_{1} C_{\infty} J_{\infty}^{q-2}+\ldots \\
\ldots+A_{q-1} C_{F} J_{F}^{q-1}+\sigma A_{q} C_{F} J_{F}^{q-1} & \ldots & \ldots+A_{q-1} C_{\infty}+\sigma A_{q} C_{\infty}
\end{array}\right] \\
& \stackrel{(83)(84)}{=}\left[\begin{array}{lll}
C_{F}\left(\sigma I_{n}-J_{F}\right) & \ldots & C_{\infty} J_{\infty}^{q-2}\left(\sigma J_{\infty}-I_{\mu}\right) \\
C_{F} J_{F}\left(\sigma I_{n}-J_{F}\right) & \ldots & C_{\infty} J_{\infty}^{q-3}\left(\sigma J_{\infty}-I_{\mu}\right) \\
\vdots & \ldots & \vdots \\
C_{F} J_{F}^{q-2}\left(\sigma I_{n}-J_{F}\right) & \ldots & C_{\infty}\left(\sigma J_{\infty}-I_{\mu}\right) \\
A_{q} C_{F} J_{F}^{q-1}\left(\sigma I_{n}-J_{F}\right) & \ldots & A_{0} C_{\infty} J_{\infty}^{q-1}+A_{1} C_{\infty} J_{\infty}^{q-2}+\ldots+A_{q-1} C_{\infty} \\
& & -\sigma A_{0} C_{\infty} J_{\infty}^{q}-\sigma A_{1} C_{\infty} J_{\infty}^{q-1}-\ldots-\sigma A_{q-1} J_{\infty} C_{\infty}
\end{array}\right] \\
& =\left[\begin{array}{lll}
C_{F}\left(\sigma I_{r}-J_{F}\right) & \ldots & C_{\infty} J_{\infty}^{q-2}\left(\sigma J_{\infty}-I_{\mu}\right) \\
C_{F} J_{F}\left(\sigma I_{r}-J_{F}\right) & \ldots & C_{\infty} J_{\infty}^{q-3}\left(\sigma J_{\infty}-I_{\mu}\right) \\
\vdots & \ldots & \vdots \\
C_{F} J_{F}^{q-2}\left(\sigma I_{r}-J_{F}\right) & \ldots & C_{\infty}\left(\sigma J_{\infty}-I_{\mu}\right) \\
A_{q} C_{F} J_{F}^{q-1}\left(\sigma I_{n}-J_{F}\right) & \ldots & -A_{0} C_{\infty} J_{\infty}^{q-1}\left(\sigma J_{\infty}-I_{\mu}\right)-A_{1} C_{\infty} J_{\infty}^{q-2}\left(\sigma J_{\infty}-I_{\mu}\right)-\ldots
\end{array}\right] \\
& =\left[\begin{array}{lll}
C_{F} & \ldots & C_{\infty} J_{\infty}^{q-2} \\
C_{F} J_{F} & \ldots & C_{\infty} J_{\infty}^{q-3} \\
\vdots & \ldots & \vdots \\
C_{F} J_{F}^{q-2} & \ldots & C_{\infty} \\
A_{q} C_{F} J_{F}^{q-1} & \ldots & -\left[A_{0} C_{\infty} J_{\infty}^{q-1}+A_{1} C_{\infty} J_{\infty}^{q-2}+\ldots+A_{q-1} C_{\infty}\right]
\end{array}\right] \\
& \times\left[\begin{array}{ll}
\sigma I_{n}-J_{F} & 0_{n, \mu} \\
0_{\mu, n} & \sigma J_{\infty}-I_{\mu}
\end{array}\right]
\end{aligned}
$$


Let now $A_{i}(\sigma)=A_{i q^{i}} \sigma^{q^{i}}+A_{i q^{i}-1} \sigma^{q^{i}-1}+\ldots+A_{i 1} \sigma+A_{i 0} \in \mathbb{R}[\sigma]^{r_{i} \times r_{i}}$, $i=1,2$ be two polynomial matrices such that $r_{1} q^{1}=r_{2} q^{2}=: p$ and let

$$
\sigma \overline{E_{i}}-\bar{A}_{i}:=\left[\begin{array}{ccccc}
\sigma I_{r_{i}} & -I_{r_{i}} & \ldots & 0 & 0 \\
0 & \sigma I_{r_{i}} & \ldots & 0 & 0 \\
\vdots & \vdots & \ddots & \vdots & \vdots \\
0 & 0 & \ldots & \sigma I_{r_{i}} & -I_{r_{i}} \\
A_{i 0} & A_{i 1} & \ldots & A_{i q^{i}-2} & \sigma A_{i q^{i}}+A_{i q^{i}-1}
\end{array}\right] \in \mathbb{R}[\sigma]^{p \times p} \quad i=1,2
$$

Definition 3 Let $0<p \in \mathbb{Z}^{+}$be a fixed and consider the set $\mathbb{R}_{p}[\sigma]$ of polynomial matrices $A_{i}(\sigma)=A_{i q^{i}} \sigma^{q^{i}}+A_{i q^{i}-1} \sigma^{q^{i}-1}+\ldots+A_{i 1} \sigma+A_{i 0} \in \mathbb{R}[\sigma]^{r_{i} \times r_{i}}$ such that $r_{i} q^{i}=p$. Two polynomial matrices $A_{i}(\sigma)=A_{i q^{i}} \sigma^{q^{i}}+A_{i q^{i}-1} \sigma^{q^{i}-1}+$ $\ldots+A_{i 1} \sigma+A_{i 0} \in \mathbb{R}_{p}[\sigma], i=1,2$ are called strictly equivalent iff the matrix pencils $\sigma \bar{E}_{1}-\bar{A}_{1} \in \mathbb{R}[\sigma]^{p \times p}$ and $\sigma \bar{E}_{2}-\bar{A}_{2} \in \mathbb{R}[\sigma]^{p \times p}$ are strictly equivalent [9], i.e. iff there exist nonsingular $M \in \mathbb{R}^{p \times p}, Q \in \mathbb{R}^{p \times p}$ such that

$$
\left[\sigma \bar{E}_{1}-\bar{A}_{1}\right]=M\left[\sigma \bar{E}_{2}-\bar{A}_{2}\right] Q
$$

If $A_{i}(\sigma) \in \mathbb{R}_{p}[\sigma], i=1,2$ are strictly equivalent then we denote this fact by witting $\left(A_{1}(\sigma), A_{2}(\sigma)\right) \in \mathcal{S E}$. Obviously $\mathcal{S E}$ is an equivalence relation on $\mathbb{R}_{p}[\sigma] \times \mathbb{R}_{p}[\sigma]$ and we have

Proposition 9 Let $A_{i}(\sigma)=A_{i q^{i}} \sigma^{q^{i}}+A_{i q^{i}-1} \sigma^{q^{i}-1}+\ldots+A_{i 1} \sigma+A_{i 0} \in \mathbb{R}_{p}[\sigma], i=$ 1,2 be strictly equivalent. Then $A_{1}(\sigma) \in \mathbb{R}[\sigma]^{r_{1} \times r_{1}}$ and $A_{2}(\sigma) \in \mathbb{R}[\sigma]^{r_{2} \times r_{2}}$ have identical finite and infinite elementary divisors.

Proof. ¿From Lemma 6 the polynomial matrix $A_{i}(\sigma)$ and the matrix pencil $\left[\sigma \bar{E}_{i}-\bar{A}_{i}\right] \in \mathbb{R}[\sigma]^{p \times p}, i=1,2$ have identical finite and infinite elementary divisors and the result follows since $A_{1}(\sigma) \in \mathbb{R}[\sigma]^{r_{1} \times r_{1}}$ and $A_{2}(\sigma) \in \mathbb{R}[\sigma]_{q_{2} r_{2}}^{r_{2} \times r_{2}}$ are strictly equivalent iff $\sigma \bar{E}_{1}-\bar{A}_{1}$ and $\sigma \bar{E}_{2}-\bar{A}_{2}$ are strictly equivalent, i.e. iff they have identical finite and infinite elementary divisors[9].

Consider now two strictly equivalent polynomial matrices $A_{i}(\sigma)=A_{i q^{i}} \sigma^{q^{i}}+$ $A_{i q^{i}-1} \sigma^{q^{i}-1}+\ldots+A_{i 1} \sigma+A_{i 0} \in \mathbb{R}_{p}[\sigma], i=1,2$, the associated discrete-time AR-representations

$$
A_{i}(\sigma) \xi_{k}^{i}=0, \quad k=0,1,2, \ldots, N-q^{i} \geq 0, \quad i=1,2
$$

and their first order realizations over the finite time interval $k=0,1,2, \ldots, N$ :

$$
\left[\sigma \bar{E}_{i}-\bar{A}_{i}\right] x_{k}^{i}=0, \quad k=0,1,2, \ldots, N-q^{i} \geq 0, \quad i=1,2
$$

so that the pencils $\left[\sigma \bar{E}_{1}-\bar{A}_{1}\right] \in \mathbb{R}[\sigma]^{p \times p}$ and $\left[\sigma \bar{E}_{2}-\bar{A}_{2}\right] \in \mathbb{R}[\sigma]^{p \times p}$ are strictly equivalent. If $M_{i} \in \mathbb{R}^{p \times p}, Q_{i} \in \mathbb{R}^{p \times p}, i=1,2$ are the matrices transforming (according to Lemma 8) each pencil $\sigma \bar{E}_{i}-\bar{A}_{i}, i=1,2$ to their (common) Weierstrass canonical form:

$$
[\sigma E-A]:=M_{1}\left[\sigma \bar{E}_{1}-\bar{A}_{1}\right] Q_{1}=M_{2}\left[\sigma \bar{E}_{2}-\bar{A}_{2}\right] Q_{2}
$$


then the transforming matrices $M \in \mathbb{R}^{p \times p}, Q \in \mathbb{R}^{p \times p}$ in (86) are given by

$$
\begin{aligned}
M & =M_{1}^{-1} M_{2} \\
Q & =Q_{2} Q_{1}^{-1}
\end{aligned}
$$

and we can state

Theorem 10 If the polynomial matrices $A_{i}(\sigma) \in \mathbb{R}_{p}[\sigma], i=1,2$ are strictly equivalent then the AR-representations

$$
A_{i}(\sigma) \xi_{k}^{i}=0, \quad k=0,1,2, \ldots, N-q^{i} \geq 0, \quad i=1,2
$$

are fundamentally equivalent over the finite time interval $k=0,1,2, \ldots N$.

Proof. Firstly and according to Proposition 9, the polynomial matrices $A_{i}(\sigma) \in$ $\mathbb{R}[\sigma]^{r_{i} \times r_{i}}, i=1,2$ will have isomorphic finite and infinite elementary divisors structure which implies that

$\operatorname{dim} \mathcal{B}_{A_{1}(\sigma)}=r_{1} q^{1}=\operatorname{dim} \mathcal{B}_{\sigma \bar{E}_{1}-\bar{A}_{1}}=r_{2} q^{2}=\operatorname{dim} \mathcal{B}_{\sigma \bar{E}_{2}-\bar{A}_{2}}=\operatorname{dim} \mathcal{B}_{A_{2}(\sigma)}=n+\mu$,

By assumption

$x_{k}^{i}:=\left[\begin{array}{l}\xi_{k}^{i} \\ \xi_{k+1}^{i} \\ \vdots \\ \xi_{k+q^{i}-2}^{i} \\ \xi_{k+q^{i}-1}^{i}\end{array}\right]=\left[\begin{array}{c}I_{r_{1}} \\ I_{r_{1}} \sigma \\ \vdots \\ I_{r_{1}} \sigma^{q^{i}-2} \\ I_{r_{1}} \sigma^{q^{i}-1}\end{array}\right] \xi_{k}^{i}=S_{r_{i} q_{i}} \xi_{k}^{i}, \quad i=1,2$ and $k=0,1,2, \ldots, N-\left(q^{i}-1\right)$

so that $\left[\sigma \bar{E}_{1}-\bar{A}_{1}\right] x_{k}^{1}=0, k=0,1,2, \ldots, N-q^{1}$ which due to (86) can be written as

$$
M\left[\sigma \bar{E}_{2}-\bar{A}_{2}\right] Q x_{k}^{1}=0, \quad k=0,1,2, \ldots, N-q^{1}
$$

which due to $\left[\sigma \bar{E}_{2}-\bar{A}_{2}\right] x_{k}^{2}=0, k=0,1,2, \ldots, N-q^{2}$ implies that $Q x_{k}^{1} \in$ $\mathcal{B}_{\sigma \bar{E}_{2}-\bar{A}_{2}}, k=0,1,2, \ldots, N-q^{1}$. Since $Q$ is square and invertible it is injective and from (92) it follows that it is surjective so that

$$
Q: \mathcal{B}_{\left[\sigma \bar{E}_{1}-\bar{A}_{1}\right]} \rightarrow \mathcal{B}_{\left[\sigma \bar{E}_{2}-\bar{A}_{2}\right]} \mid x_{k}^{1} \mapsto x_{k}^{2}=Q x_{k}^{1}, \quad k=0,1,2, \ldots, N-q^{1}
$$

is the bijection between the respective behaviors. Now from Theorem 7

$$
\xi_{k}^{2}=\left[\begin{array}{llll}
I_{r_{2}} & 0 & \ldots & 0
\end{array}\right] x_{k}^{2}
$$

and $L_{r_{2} q_{2}}(\sigma):=\left[\begin{array}{llll}I_{r_{2}} & 0 & \ldots & 0\end{array}\right]$ is the bijection $\mathcal{B}_{\left[\sigma \bar{E}_{2}-\bar{A}_{2}\right]} \rightarrow \mathcal{B}_{A_{2}(\sigma)} \mid x_{k}^{2} \mapsto$ $\xi_{k}^{2}$ while

$$
x_{k}^{1}=S_{r_{1} q_{1}}(\sigma) \xi_{k}^{1}
$$

and $S_{r_{1} q_{1}}(\sigma)$ is the bijection $\mathcal{B}_{A_{1}(\sigma)} \rightarrow \mathcal{B}_{\left[\sigma \bar{E}_{1}-\bar{A}_{1}\right]} \mid \xi_{k}^{1} \mapsto x_{k}^{1}$. Combining (95)(94) and (96) we have

$$
\begin{aligned}
\xi_{k}^{2} & =\left[\begin{array}{llll}
I_{r_{2}} & 0 & \ldots & 0
\end{array}\right] x_{k}^{2} \\
& =\left[\begin{array}{llll}
I_{r_{2}} & 0 & \ldots & 0
\end{array}\right] Q x_{k}^{1} \\
& =\left[\begin{array}{llll}
I_{r_{2}} & 0 & \ldots & 0
\end{array}\right] Q S_{r_{1} q_{1}}(\sigma) \xi_{k}^{1}
\end{aligned}
$$


Since the maps in (95)(94) and (96) are all bijective their composition in (97) is bijective and thus the bijective polynomial map between the behaviors $\mathcal{B}_{A_{1}(\sigma)}$ and $\mathcal{B}_{A_{2}(\sigma)}$ is

$$
Q_{12}(\sigma): \mathcal{B}_{A_{1}(\sigma)} \rightarrow \mathcal{B}_{A_{2}(\sigma)} \mid \xi_{k}^{1} \mapsto \xi_{k}^{2}
$$

where

$$
Q_{12}(\sigma)=\left[\begin{array}{llll}
I_{r_{2}} & 0 & \ldots & 0
\end{array}\right] \underset{p \times p}{Q}\left[\begin{array}{c}
I_{r_{1} \times p} \\
I_{r_{1}} \sigma \\
\vdots \\
I_{r_{1}} \sigma^{q^{1}-2} \\
I_{r_{1}} \sigma^{q^{1}-1} \\
p \times r_{1}
\end{array}\right] \in \mathbb{R}[\sigma]^{r_{2 \times} r_{1}}
$$

Similar arguments prove that the inverse bijective polynomial map between $\mathcal{B}_{A_{2}(\sigma)}$ and $\mathcal{B}_{A_{1}(\sigma)}$ is

$$
Q_{21}(\sigma): \mathcal{B}_{A_{2}(\sigma)} \rightarrow \mathcal{B}_{A_{1}(\sigma)} \mid \xi_{k}^{2} \mapsto \xi_{k}^{1}
$$

where

$$
Q_{21}(\sigma)=\left[\begin{array}{llll}
I_{r 1} & 0 & \ldots & 0
\end{array}\right] \underset{p \times p}{Q_{r_{1 \times p}}^{-1}}\left[\begin{array}{c}
I_{r_{2}} \\
I_{r_{2}} \sigma \\
\vdots \\
I_{r_{2}} \sigma^{q^{2}-2} \\
I_{r 2} \sigma^{q^{2}-1} \\
p \times r 2
\end{array}\right] \in \mathbb{R}[\sigma]^{r_{1 \times} r_{2}}
$$

and thus according to definition 1 the AR-representations in (91) are fundamentally equivalent over the finite time interval $N$. (see diagram bellow)

$$
\begin{array}{ccc}
\mathcal{B}_{A_{1}(\sigma)} & \stackrel{Q_{21}(\sigma)}{\longleftarrow} & \mathcal{B}_{A_{2}(\sigma)} \\
L_{r_{1} q_{1}}(\sigma) \uparrow \downarrow S_{r_{1} q_{1}}(\sigma) & \stackrel{Q_{12}(\sigma)}{\longrightarrow} & S_{r_{2} q_{2}}(\sigma) \downarrow \uparrow L_{r_{2} q_{2}}(\sigma) \\
\mathcal{B}_{\left[\sigma \bar{E}_{1}-\bar{A}_{1}\right]} & \stackrel{Q^{-1}}{\rightleftarrows} & \mathcal{B}_{\left[\sigma \bar{E}_{2}-\bar{A}_{2}\right]}
\end{array}
$$

\section{Conclusions}

We have presented some new algebraic properties of nonsingular polynomial matrices that although seem to be obvious they have not appear explicitly anywhere in the literature. These results concern the structure of the elementary divisors of a nonsingular polynomial matrix and its relation to the corresponding structure of the block companion matrix in (7). As a direct application we have proposed a first order realization method for discrete time AR-representations which requires only a few trivial manipulations. Notice here that unlike fundamental equivalence defined for continuous time systems [18][6] which requires 
isomorphic zero structures (finite and infinite) of the polynomial matrices involved, the proposed equivalence requires isomorphic elementary divisors structure (finite and infinite), which in general are more than zeros (with multiplicities accounted for). This can be partially justified by the fact that we consider discrete time systems over finite time intervals. As a consequence the values of $\xi_{k}$ at both end points of the interval (i.e. initial and final values) are employed as arbitrary parameters. It is thus expected that the dimension of a realization of an AR-representation in discrete time will be in general greater than the corresponding continuous time one.

A very interesting and open problem which follows from the above analysis is that of the algebraic characterization of the equivalence relation between strictly equivalent polynomial matrices, i.e. between polynomial matrices $A_{i}(\sigma)=A_{i q^{i}} \sigma^{q^{i}}+A_{i q^{i}-1} \sigma^{q^{i}-1}+\ldots+A_{i 1} \sigma+A_{i 0} \in \mathbb{R}[\sigma]^{r_{i} \times r_{i}}, i=1,2$ with $r_{1} q^{1}=r_{2} q^{2}$ having isomorphic finite and infinite elementary divisors structures as the ones in the second example above.

\section{References}

[1] Luenberger D.G.,Dynamic Equations in Descriptor Form, IEEE Trans .Autom. Contr., Vol-22, No 3, June 1977, pp. 312-321.

[2] Luenberger D.G., Boundary Recursion for Descriptor Variable Systems, IEEE Trans .Autom. Contr., Vol-34, No 3, March 1989, pp. 287-292.

[3] Lewis F. L., Descriptor Systems: Decomposition into Forward and Backward Subsystems, IEEE Trans .Autom. Contr., Vol-29, No 2, February 1984, pp. 167-170.

[4] Lewis F. L., B.G.Mertzios, On the Analysis of Discrete Linear TimeInvariant Singular Systems, IEEE Trans. Autom. Contr., Vol 35, No 4, April 1990, pp.506 - 511.

[5] Lewis F. L., A Survey of Linear Singular Systems, Circuits Systems and Signal Processing, Vol 5, No 1, 1986, pp.3 - 36.

[6] A.C. Pugh, E. Antoniou, N. Karampetakis, G. Hayton, Fundamental equivalence of non-regular AR-representations, submitted to Automatica, May 1998.

[7] E. Antoniou, A.I.G. Vardulakis, N. Karampetakis, A spectral characterization of the behavior of discrete time AR-representations over a finite time interval, Kybernetika, vol. 34 (1998), No 5, pp. 555-564.

[8] Nikoukhah R, Willsky A., Bernard C. Levy, Boundary-value descriptor systems: well-posedness, reachability and observability, Int. J. Control, 1987, Vol 46, 1715 - 1737.

[9] Gantmacher F.R., Matrix Theory, Chelsea Publishing Company, 1971, New York.

[10] Gohberg I., Lancaster P., Rodman L., Matrix Polynomials, Academic Press Inc., 1982, New York. 
[11] Gohberg I., Kaashoek M.A., Lerer L., Rodman L., Common Multiples and Common Divisors of Matrix Polynomials, I. Spectral Method, Indiana Journal of Math. 30, 1981, 321-356.

[12] Gohberg I., Kaashoek M.A., Lerer L., Rodman L., Common Multiples and Common Divisors of Matrix Polynomials, II. VanderMonde and Resultant Matrices, Linear and Multilinear Algebra, 1982, Vol 12, pp. 159 - 203.

[13] Vardulakis A.I.G., Linear Multivariable Control - Algebraic Analysis and Synthesis Methods, John Willey \& Sons Ltd, 1991, New York.

[14] Vardulakis A.I.G, Karcanias N., Relations between strict equivalence invariants and structure at infinity of matrix pencils, IEEE Trans. on Autom. Control, Vol. AC-28, No.4, April 1983.

[15] Vardulakis A.I.G. and Antoniou S., On the solution and impulsive behaviour of polynomial matrix descriptions of free linear multivariable systems, Int.J. Control, 1999, Vol. 72, No.3, 215-228.

[16] Vardulakis A.I.G. and Fragulis G., Infinite elementary divisors of polynomial matrices and impulsive solutions of linear homogeneous matrix differential equations, Circuits, Systems and Sygnal Process, 1989, 8, 357-373.

[17] Antoniou E.N, Analysis of linear discreete time singular systems, Ph.D. Thesis, Department of Mathematics, Aristotle University of Thessalioniki, Thrssaloniki, Greece, 2000.

[18] Pugh, A.C., Karampetaks,N.P., Vardulakis, A.I.G. and Hayton, G.E., A funddamental equivalence notion for Linear multivariable systems, IEEE Trans. on Autom. Control, Vol. AC-39, No.5, May 1994. 